\title{
Polysaccharides from ophiopogon japonicus protect against oxidative damage induced by strenuous exercise
}

\author{
Dahu Sun. Jinyang Lin ${ }^{\mathrm{a}}$ \\ Department of Physical Education, Central South University, Changsha City, Hunan Province, 410083, China
}

\begin{abstract}
Dry tuberous roots of ophiopogon japonicus are famous Chinese herbal medicines. Several studies have been shown that polysaccharide is one of the main active ingredients of ophiopogon japonicus and plays an important role in pharmacological action. In the study, the effects of polysaccharides from ophiopogon japonicus (POJ) on the oxidative damage induced by strenuous exercise were investigated. Male mice were randomly divided into four groups and designated as high-dose POJ-treated, medium-dose POJ-treated, low-dose POJ-treated and control groups, which received 80, 40, and $20 \mathrm{mg} / \mathrm{kg}$ POJ and physiological saline, respectively, by gavage once a day for 28 days. This was followed by an exhaustive running exercise and measurements of various biochemical parameters from blood and muscles tissue. The results showed that POJ could significantly prolong the exhaustive running time of mice, decrease the levels of lactate dehydrogenase (LDH), creatine kinase (CK) and 8-Hydroxy-2'-deoxyguanosine (8-OHdG) in blood, and malondialdehyde (MDA) levels in muscle, which were accompanied by corresponding increased levels of superoxide disproportionation enzyme (SOD), glutathione peroxidase (GPx), and catalase (CAT) in muscle. The data suggest that POJ have protective effects on strenuous exercise-induced oxidative damage.
\end{abstract}

\section{Introduction}

Ophiopogon japonicus (L. F) Ker-Gawl is a perennial herb of the genus Liliaceae, which distributes in Japan, Vietnam, India and southern China, such as Guangdong, Guangxi, Fujian, Taiwan, Zhejiang, Jiangsu and many more places [1]. Dry tuberous roots of ophiopogon japonicus (known as MaiDong) are famous Chinese herbal medicines, which have the effect of quenching thirst, moistening lungs and relieving cough. Recent literature has reported that ophiopogon japonicus extracts have anti-oxidation, anti-fatigue, anti-arrhythmia, hypoglycemic, cellular immune function and recovery of islet cell function [2-4]. The main chemical components of Ophiopogon japonicus are polysaccharides, steroidal saponins, amino acids, etc. Polysaccharide is one of the main active ingredients of ophiopogon japonicus and plays an important role in pharmacological action [5].

At present, there are few studies on the effects of polysaccharides from ophiopogon japonicus (POJ) on oxidative damage caused by strenuous physical exercise. Therefore, this study used exhaustive exercised-mouse model to study the protective effects of POJ on strenuous exercise-induced oxidative damage by measuring the changes of various biochemical parameters, which would provide a theoretical basis for the development of POJ as a related exercise supplement to improve the body's oxidative stress.

\section{Materials and methods}

\subsection{Plant material}

Ophiopogon japonicus were purchased from the local Chinese herbal medicine market, and were identified by botanist Prof. M.C. Wan (School of Life Sciences, Hunan Normal University, Changsha, China). Ophiopogon japonicus were washed with water, and dried in an oven at $65{ }^{\circ} \mathrm{C}$, then pulverized with a fine powder, sieved through $40 \mathrm{mesh}$, and fine powder was stored at $4{ }^{\circ} \mathrm{C}$ until further use.

\subsection{Chemicals and reagents}

Assay kits for lactate dehydrogenase (LDH), creatine kinase (CK) assay kit, and malondialdehyde (MDA) were purchased from JianCheng Biotech Co., Ltd. (NanJing, China). Assay kits for superoxide disproportionation enzyme (SOD), glutathione peroxidase (GPx), and catalase (CAT) were purchased from LanJi Biotechnology Co., Ltd. (ShangHai, China). 8-Hydroxy-2'-deoxyguanosine (8-OHdG) enzyme-linked immunosorbent assay kit was purchased from AiMeiJie Technology Co., Ltd. (WuHan, China). Other reagents were of analytical grade and purchased from Hunan Reagent Co. (ChangSha, China) .

\footnotetext{
${ }^{a}$ Corresponding author: linjinyangzn@126.com
} 


\subsection{Preparation of polysaccharides from ophiopogon japonicus}

The ophiopogon japonicus powders were refluxed twice with $80 \%$ ethanol at $80{ }^{\circ} \mathrm{C}$ to remove some of the lipids and pigments, and the ethanol is recovered. 10 times of water was added to the residue and boil for 3 times, each time for $1 \mathrm{~h}$. After centrifugation, the filtrates were combined, concentrated under reduced pressure, and deproteinized by Sevag method. The concentrate was added to 4 times $95 \%$ ethanol overnight, and the precipitate was collected after centrifugation, washed successively with absolute ethanol, acetone, petroleum ether, and then dialyzed in a dialysis bag for $24 \mathrm{~h}$, precipitated with absolute ethanol, and vacuum was dried to obtain ophiopogon japonicus polysaccharide (POJ).

\subsection{Animals and grouping}

Male Kunming mice, each weighing $(18 \pm 2) \mathrm{g}$, were provided by the Laboratory Animal Center of Hunan (Changsha, China). Animals were tested after 7 days of adaptation to the laboratory environment. The mice had unrestricted access to drinking water and pellet diet (based on national standard rodent feed configuration). The environment of the animal breeding room is as follows: temperature of $21-25{ }^{\circ} \mathrm{C}$, relative humidity of $40-60 \%, 12 \mathrm{~h} \mathrm{light}$ and dark alternate (9:00 a.m.-21:00 p.m.). All animals did not have the running exercise before the experiment. This experiment was approved by the Institutional Animal Ethics Committee of Central South University and was conducted in accordance with the Guiding Opinions on Treating Experimental Animals issued by the Ministry of Science and Technology of Chin.

64 mice were randomly divided into 4 groups according to body weight of sixteen mice each, namely high-dose POJ-treated (HPOJ) group, medium-dose POJ-treated (MPOJ) group, low-dose POJ-treated (LPOJ) group, and control (C) group. HPOJ group were intragastrically administered with $80 \mathrm{mg} / \mathrm{kg}$ POJ. MPOJ group were intragastrically administered with $40 \mathrm{mg} / \mathrm{kg}$ POJ. LPOJ group were intragastrically administered with $20 \mathrm{mg} / \mathrm{kg}$ POJ. C group were intragastrically administered with physiological saline $(1.0 \mathrm{~mL})$. Animals were treated once a day for 28 consecutive days.

\subsection{Running exercise}

On the 21th day, the mice in all the groups were subjected to adaptive running exercise $(10 \mathrm{~m} / \mathrm{min}, 10$ min) on a treadmill. The treadmill (JiangSu Cyrus Biotechnology Co., Ltd., China) has eight runways with a length of $60 \mathrm{~cm}$ and a speed control range of $0-100$ $\mathrm{m} / \mathrm{min}$. It uses DC constant current and current stimulation to keep the animals moving. On the 28th day, the mice were performed an incremental graded running exercise to exhaustion. In the first stage, the running speed and time of mice was $10 \mathrm{~m} / \mathrm{min}$ and $15 \mathrm{~min}$. In the second stage, the running speed and time of mice was 20 $\mathrm{m} / \mathrm{min}, 15 \mathrm{~min}$. In the third stage, mice were required to run to exhaustion at a speed of $40 \mathrm{~m} / \mathrm{min}$. The point of exhaustion was determined when the running speed of mice decreased, and they were unable to maintain the front $20 \mathrm{~cm}$ on the runway more than 3 times. Even if electrical stimulationwas given, the drive to the mice was invalid [6].

\subsection{Biochemical analysis}

After the exhaustive running exercise, the mice in all the groups were anesthetized and sacrificed immediately. Blood was collected for measurement of the levels of $\mathrm{CK}, \mathrm{LDH}$, and $8-\mathrm{OHdG}$. Then the quadriceps muscle was immediately removed, washed in physiological saline, blotted dry with filter paper, weighed, and then made into a $10 \%$ homogenate. Centrifuge at $4{ }^{\circ} \mathrm{C}$ and separate the supernatant for measuring the levels of SOD, GPx, CAT, and MDA. The test method and calculation formula were strictly in accordance with the instruction manual of the kits.

\subsection{Statistical analysis}

Each group of experimental data was expressed as mean $\pm \mathrm{SD}$, and statistical analysis was performed using SPSS 15.0. One-way analysis of variance (One Way ANOVA) was used for the difference between groups. The values of $p<0.05$ were considered as significant.

\section{Results and Discussion}

\subsection{Effect of POJ on exhaustion running time of mice}

The decline in exercise endurance is the most important manifestation of fatigue, and it is also the most direct and objective indicator of fatigue [7]. Exercise endurance measurement methods include treadmill exercise, climbing exercise and swimming exercise. Treadmill exercise can replace traditional swimming exercise, which makes the exercise intensity index more accurate, and is one of the necessary methods to exercise endurance and exercise injury [8]. In this study, the treadmill exercise test was used to evaluate the exercise endurance of POJ in mice.

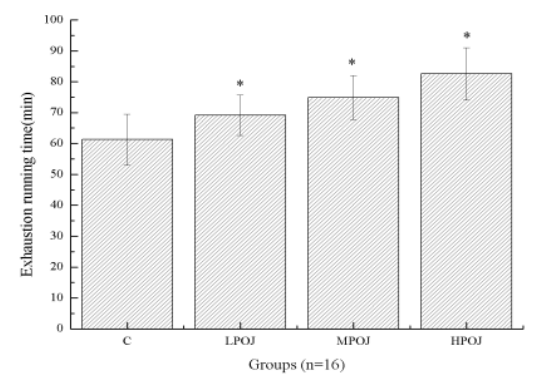

Figure 1. Effect of POJ on exhaustion running time of mice. Data were presented as means \pm SD. ${ }^{*} \mathrm{p}<0.05$ as compared with the control (C) group. 
As shown in Figure 1, compared with the C group, the exhaustive running times of HPOJ, MPOJ, and LPOJ groups were significantly prolonged $(p<0.05)$. These results clearly indicated that different doses of POJ can improve exercise endurance and delay exercise-induced fatigue in mice.

\subsection{Effect of POJ on antioxidant enzymes levels in muscle of mice}

SOD, CAT, and GPx are the first line of defense for the body's antioxidant enzymes system to eliminate ROS during strenuous exercise. SOD could catalyze $\mathrm{O}_{2}$ - to make it disproportionate to form $\mathrm{O}_{2}$ and $\mathrm{H}_{2} \mathrm{O}_{2}$. CAT could catalyze the decomposition of $\mathrm{H}_{2} \mathrm{O}_{2}$ into $\mathrm{O}_{2}$ and $\mathrm{H}_{2} \mathrm{O}$. GPx could catalyze glutathione (GSH) into oxidized glutathione (GSSG), reduce toxic peroxides to non-toxic hydroxy compounds, thereby protecting the structure and function of the cell membrane from the interference and damage of the peroxide [9].

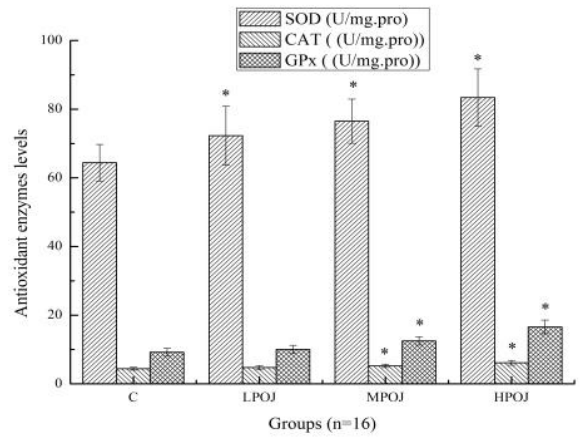

Figure 2. Effect of POJ on antioxidant enzymes levels in muscle of mice. Data were presented as means \pm SD. ${ }^{*} p<0.05$ as compared with the control $(\mathrm{C})$ group.

As shown in Figure 2, compared with the C group, the SOD levels in muscle of HPOJ, MPOJ, and LPOJ groups, as well as the levels of GPx and CAT in muscle of HPOJ and MPOJ groups, were significantly increased $(p<0.05)$. These results clearly indicated that medium and high doses of POJ could significantly increase the activities of SOD, CAT and GPx in muscle tissue of mice after strenuous exercise. The important antioxidant effect of POJ in protective strenuous exercise-induced oxidative stress might be related to the destruction of free-radical chain reactions, and the specific reasons need to be further studied.

\subsection{Effect of POJ on MDA levels in muscle of mice}

Lipid peroxidation (LPO) refers to the oxidative deterioration of polyunsaturated fatty acids and lipids. LPO could cause severe damage to cell membranes, lipoproteins, and other lipid-containing structures, such as altering the fluidity and permeability of cell membranes, damaging DNA and proteins, and affecting the normal function of cells [10]. MDA is the most abundant end product of LPO and its level is a key marker of LPO. Relevant research has confirmed that strenuous exercise can cause oxidative damage by increasing the amount of LPO in tissues and blood [11].

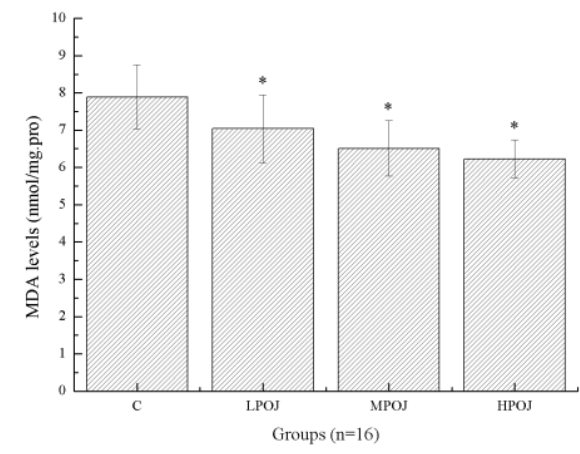

Figure 3. Effect of POJ on MDA levels in muscle of mice. Data were presented as means \pm SD. ${ }^{*} p<0.05$ as compared with the control $(\mathrm{C})$ group.

As shown in Figure 3, compared with the $\mathrm{C}$ group, the MDA levels in muscle of HPOJ, MPOJ, and LPOJ groups were significantly decreased $(p<0.05)$. These results clearly indicated that POJ could reduce LPO and delay ROS-induced oxidative stress after strenuous exercise.

\subsection{Effect of POJ on the levels of LDH and CK in blood of mice}

LDH is an important enzyme in the carbohydrate anaerobic metabolism in the body. Under normal circumstances, changes in blood LDH levels reflect the degree of lactic acid metabolism. Strenuous exercise could cause tissue damage or necrosis, such as skeletal muscle, myocardium, etc., and enhanced cell membrane permeability, leading to increased LDH leakage into the blood and increased LDH levels in the blood [12]. CK is a large molecular protein that cannot enter the blood circulation system directly. CK could leak after the cells were damaged, and it enters the lymph fluid through the interstitial space, and finally enters the blood circulation. Therefore, cell damage could be determined by measuring CK level [13]. Therefore, blood LDH and CK are two sensitive indicators of exercise-induced skeletal muscle damage.

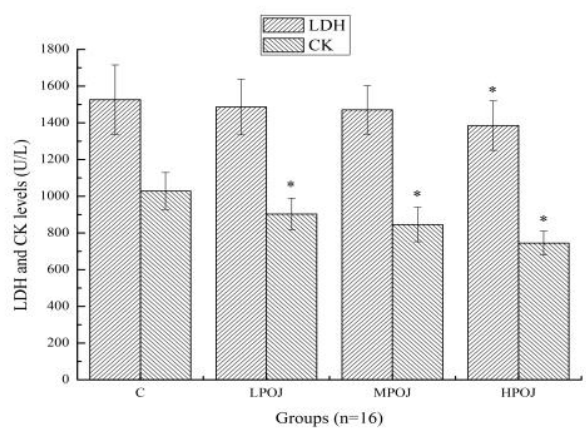

Figure 4. Effect of POJ on the levels of LDH and CK in blood of mice. Data were presented as means \pm SD. ${ }^{*} p<0.05$ as compared with the control (C) group. 
As shown in Figure 4, compared with the C group, the LDH levels in blood of HPOJ group, as well as the CK levels in blood of HPOJ, MPOJ, and LPOJ groups, were significantly decreased $(p<0.05)$. These results clearly indicated that POJ could reduce the oxidative damage of skeletal muscle induced by strenuous exercise.

\subsection{Effect of POJ on 8-OHdG levels in blood of mice}

Organisms have developed complex enzymatic or nonenzymatic antioxidant defense systems to protect cells from damage during evolution. If the rate of ROS production exceeds the body's antioxidant capacity, oxidative stress would occur [14]. More than $90 \%$ of the oxygen in cells is consumed in mitochondria. Mitochondria could produce ROS during aerobic metabolism, and mitochondrial DNA damage could occur when ROS are accumulated in the mitochondria. $8-\mathrm{OHdG}$ is one of the more than 20 major modification products of oxidative DNA damage caused by ROS. It is mainly formed by a large amount of ROS attacking the C-8 on guanine or deoxyguanosine in DNA [10]. Therefore, $8-\mathrm{OHdG}$ is a better biomarker to reflect the DNA oxidative damage.

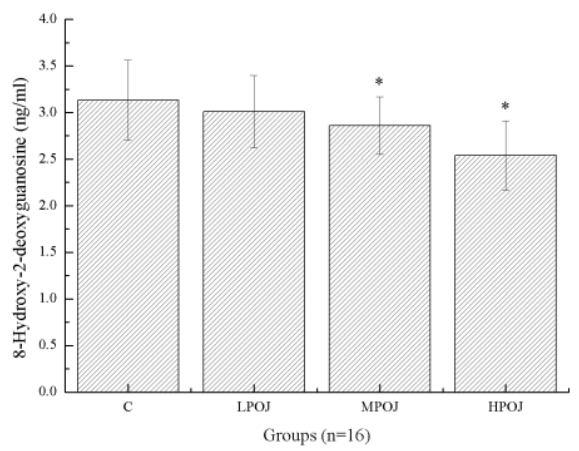

Figure 5. Effect of POJ on 8-OHdG levels in blood of mice. Data were presented as means $\pm \mathrm{SD}$. ${ }^{*} \mathrm{p}<0.05$ as compared with the control (C) group.

As shown in Figure 5, compared with the $\mathrm{C}$ group, the 8-OHdG levels in blood of HPOJ and MPOJ groups were significantly decreased $(\mathrm{p}<0.05)$. These results clearly indicated that POJ has a better repair effect on DNA oxidative damage.

\section{Summary}

The experimental data demonstrates that POJ could significantly prolong the exhaustive running time of mice; decrease the levels of $\mathrm{LDH}, \mathrm{CK}$ and 8-OHdG in blood, and MDA levels in muscle, which were accompanied by corresponding increase the levels of SOD, GPx, and CAT in muscle. These results suggest that POJ have protective effects on strenuous exerciseinduced oxidative damage. Further research should analyze the molecular mechanism of POJ against oxidative damage.

\section{References}

1. M.H. Chen, X.J. Chen, M. Wang, L.G. Lin, Y.T. Wang, J. EthnopharmacoL. 181 (2016)

2. M. Zhao, W.F. Xua, H.Y. Shen, P.Q. Shen, D.D. Wang, H. Xu, H.Wang, T.T.Yan, L.Wang, H.P. Hao, G.J. Wang, L. Cao, J. Pharmaceut. BIomed 138 (2017)

3. X.M. Chen, J. Jin, J. Tang, Z.F. Wang, J.J. Wang, L.Q. Jin, J.X. Lu, Carbohyd. Polym. 83 (2011)

4. C.T. Horng, H.Y. Wang, Y.S. Huang, J.S. Yang, F.A. Chen, F.A. Chen, Int. J. Pharm. Chem. Sci. 3 (2014)

5. X. Wang, R. Sun, J. Zhang, Y. Chen, N. Liu, Fitoterapia. 83 (2012)

6. G.X Fan, Agro Food Industry Hi-Tech. 28 (2017)

7. F.Miao, D. Wu, G. Ni, Afr .J. Tradit. Complem. 13 (2016)

8. Y.F, Xu, Int. J. Med. Mushrooms. 18 (2016)

9. R. Guo, B. Qi, Trop. J. Pharm. Res. 13 (2014)

10. J.C. He, R.W. Li, H.Y. Zhu, Biomed. Res-India. 181 (2017)

11. C.C. Huang, W.C. Huang, S.C. Yang, C.C. Chan, W.T.Lin, Molecules. 18 (2013)

12. T. Art, H. Amory, D. Desmecht, P. Lekeux, Equine. Ve.t J.. 181 (2016)

13. H Kuipers, Int. J. Sports Med. 15 (1994)

14. N. N. Xiao, Biomol Ther. 23 (2015) 